ISSN 2226-4787

www.mdpi.com/journal/pharmacy

Article

\title{
Self-Directed Online Learning Modules: Students' Behaviours and Experiences
}

\section{Stephen Maloney *, Joanna Hong-Meng Tai, Sophie Paynter, Kristin Lo and Dragan Ilic}

School of Primary Health Care, Monash University Physiotherapy, Monash University, PO Box 527, Frankston, Vic, Melbourne 3199, Australia; E-Mails: joanna.tai@monash.edu (J.H.-M.T.); sophie.paynter@monash.edu (S.P.); kristin.lo@monash.edu (K.L.); dragan.ilic@monash.edu (D.I.)

* Author to whom correspondence should be addressed; E-Mail: Stephen.maloney@monash.edu; Tel.: +61-3-9904-4240; Fax: +61-3-9904-4812.

Received: 2 May 2013; in revised form: 15 July 2013 / Accepted: 18 July 2013 /

Published: 29 July 2013

\begin{abstract}
Academic programs are experiencing pressure to produce efficiencies in education by incorporating online learning. Simply transplanting a text-based resource to an online setting by itself does not necessarily provide an effective online learning environment. In collaboration with clinical educators, our academic team constructed an online learning activity designed to reinforce allied health student knowledge of pharmacology. However, anecdotal reports from students entering the clinical environment indicated poor engagement with the online resources. These reports prompted the need for a method of formal analysis of student engagement and insights into student preferences and behaviours with online learning. Through auditing online usage data and survey analysis, our study determines a set of practical recommendations for the design of online resources, and their implementation, in order optimise their utilization and educational value.
\end{abstract}

Keywords: e-Learning; pedagogy; web-based; technology; self-directed

\section{Introduction}

The term self-directed learning encompasses both the external educational environment and also the internal characteristics of individuals [1,2]. The ability to be a life-long, self-directed learner is vital for health professionals, with clinicians now expecting new graduates to possess self-directed learner attributes [3]. 
Advances in the application of emerging technologies have led to greater incorporation of web-based teaching and learning activities into tertiary education [4]. Web-based delivery has influenced the learner's ability to access self-directed learning materials, and with the use of tracking and user-analysis software, it has also brought with it the ability for academics to monitor the degree of student interaction with these activities.

Self-directed learning in an online environment may assist in supporting student learning and performance during the transition to clinical education where access to university staff and learning opportunities may be diminished [5,6]. It may allow for university staff to influence the learning opportunities available to students to prevent the potential divide in competence created by the heterogeneity of clinical learning environments [7,8]. Self-directed learning may also offer flexibility of learning opportunities on campus to accommodate greater student numbers and pressure on teaching and learning resources in modern university curricula $[9,10]$.

Making course resources available online can be a great advantage, especially when distance is an issue [11], however online learning resources are not a panacea for all issues relating to efficiency and student engagement. Hung (2010) identified that, for an online self-directed learning environment, there were two major components required for learners to effectively partake: familiarity with and the ability to operate technology, and being able to self-manage their learning [12]. Simply transplanting a text-based resource to an online setting by itself does not provide an effective online learning environment [13].

At Monash University, physiotherapy students utilise knowledge gained in preclinical semesters during their clinical placements in the final two years of the four-year program. Clinical educators had reported to the academic staff that pharmacology knowledge was an identified growth area for competent clinical practice. In response, academic staff created four self-directed quiz activities, progressing from basic pharmacological actions and principles to more complex clinical scenarios. These resources were promoted as optional resources to support clinical-practice. Students could utilise these self-directed learning modules to increase their awareness of the strengths and deficits in their knowledge and allow targeted revision to improve their clinical competence. With an online learning platform already well established, academic staff placed these self-directed modules online to allow increased flexibility in time and location of access. However, a large proportion of students chose not to utilise these resources. The lack of student participation prompted academic staff to question the design and construction of these resources, triggering an evaluation of the student behaviours and perceptions of this self-directed resource.

The primary aim of this evaluation was to determine a set of recommendations, from the Monash experience, for optimising the utilisation and educational value of online self-directed learning activities. The secondary aim was to improve the learning support available to students in their transition from novice to expert clinician, through a deeper understanding of student experiences and utilisation of an online self-directed learning module.

\section{Methods}

\subsection{Participants}

Third year physiotherapy students completing their last campus based unit in semester one, and their first clinical education based unit in semester two were invited to participate in the study by 
allowing access to their usage data throughout the academic year, and contribute to the anonymous survey data on their experiences.

\subsection{Activity Design}

Student interaction with four online self-directed learning modules was observed, with each module increasing in the complexity and competency of pharmacology knowledge required to complete the quiz: (1) titled "basic terminology and principles of pharmacology", (2) "classes of drug action and mechanisms of action", (3) "medication use and varieties", and (4) "clinical decision making (applied scenarios)".

Students interacted with the online self-directed learning modules via the online learning system "Blackboard". At the beginning of semester 1, year three, students were informed on how to access the quizzes, their purpose, and the clinical relevance of the resources by way of sharing the feedback staff had received from clinicians indicating that the previous student cohort they had encountered lacked strong skills in applying pharmacology knowledge to live clinical situations. Students were also informed that the modules were not compulsory to complete, but were there to help identify and address knowledge deficits in the topic area. The modules were not supervised by staff, and no staff involvement in the modules was scheduled, although staff were happy to clarify any information or answer any student questions if the need arose.

At the completion of the module, students received immediate automated feedback indicating their performance, such as their grade for the questions undertaken, along with the answers to each question, and reasoning as to why that answer was correct. Students were able to work collaboratively if they chose to.

\subsection{Data Collection}

The study was of a mixed method design. A retrospective audit of student engagement with online modules, and a prospective survey utilizing open text and Likert scale responses to gain deeper insights into explaining the students' degree of engagement.

Quantitative audit data on students' module usage was collected by the online learning system “Blackboard". The online system logs students' access times, duration of interactions and grades for any completed quiz activities.

Separate to the collection of online usage data, participants took part in an anonymous survey aimed at capturing their perceptions of their experiences with the online module and insights into the low levels of participation. Students were asked to indicate their level of agreement with listed statements using a five point Likert scale, ranging from strongly disagree to strongly agree. The statements were, (1) the self-directed learning activities assisted me to evaluate my current strengths/weaknesses, and (2) the self-directed learning activities were more effective than self-reflection in evaluating strengths and weaknesses in my knowledge or performances. Students were also asked, "if the resources had assisted you in identifying weaknesses in knowledge or performance, did you re-directed your study to address the identified weakness?" and "did you re-take the quiz at a later date to assess your change in knowledge?" The survey concluded by asking the students "what factors would make you more likely to undertake the online self-directed learning modules?" No other questions were asked within the survey. As no 
pre-existing validated surveys met the needs of the research question, a survey was created by the authorship team, without measured content-validity. Our operational definition of "self-directed" was that the students undertake the learning activity without supervision or formal guidance. Student engagement was the voluntary willingness to undertake the learning activities, measured through accessing and completing the learning resources provided.

Ethics approval was obtained by the Monash University Human Research Ethics Committee, approval number CF10/0255-2010000099.

\subsection{Analysis}

Descriptive statistics were utilised for analysing quantitative data including student usage data and Likert scale responses ( $1=$ strongly disagree, $2=$ disagree, $3=$ neutral, $4=$ agree, $5=$ strongly agree) . Open test responses were collated and were assessed using content analysis by two authors. Common themes from each of the independent analyses were collated and discussed, before conclusion on the final themes was achieved [14].

\section{Results}

\subsection{Online Usage Data}

Audit data logged by the online learning system "Blackboard" identified that 32 of 51 (63\%) of students completed at least one pharmacology self-directed learning module throughout the year [n(\%), $3(6 \%)$ completed one module only, 7 (14\%) completing two modules only, 7 (14\%) completing three modules only, and 15 (29\%), completing all four modules available]. Student activity mainly occurred on-campus, prior to clinical placements, however the web-based resources continued to be accessed during the students' clinical placements.

\subsection{Student Survey}

Of the 51 students within the cohort, 15 (30\%) surveys were returned.

Two central themes emerged as to why students limited their engagement with the self-directed online learning modules, (1) insufficient time and (2) differing study priorities. Students remarked that:

"Some [modules] were incredibly long and time consuming."

"Did not prioritise them for clinical revision and in the end I ran out of time."

One student pointed out they did not have access to the Internet at their rural placement accommodation in order to access the online material.

The majority of students who participated in the survey agreed or strongly agreed that the quizzes helped them identify strengths and weaknesses in learning (strongly agree $=14 \%$, agree $=72 \%$, neutral $=14 \%$, disagree $=0 \%$, strongly disagree $=0 \%$ ). However, students felt more neutral about selfdirected activities being better than reflection for learning (strongly agree $=14 \%$, agree $=43 \%$, neutral $=29 \%$, disagree $=14 \%$, strongly disagree $=0 \%$ ).

Of the 15 respondents who identified an area of weakness in their knowledge from completing a module, $27 \%$ reported that they failed to act upon addressing the known knowledge deficit, and $66 \%$ 
of respondents noted that they did not re-attempt a quiz after receiving feedback on performance. The reasons for this included: already knowing the answers from completing the quiz initially, a lack of time, and differing study priorities.

Table 1 documents suggestions given by students to increase engagement with the self-directed learning modules.

Table 1. Common student recommendations for improving student engagement in the self-directed online activities.

\begin{tabular}{ll}
\hline \multicolumn{1}{c}{$\begin{array}{c}\text { Themes presented by students for } \\
\text { improving student engagement }\end{array}$} & Illustrating Participant Quote \\
\hline More time efficient & "not take too much time", "not as time consuming" \\
More enjoyable & "if they were funner[sic]?" \\
Cover a wider range of topics & "A revision section for each semester with multiple parts so \\
Contained more clinically based scenarios & you can test yourself especially before exams" \\
\hline
\end{tabular}

\section{Discussion}

Usage patterns matched McNulty (2009) where the majority of students used resources sparingly while a few students used all the resources numerous times [15]. Peaks in resource use before exams may indicate students undertaking revision prior to exams, to self-test their knowledge and identify strengths and weaknesses for revision.

Uptake of the constructed self-directed resource by students was low. The strongest survey themes emerging for explaining the low uptake were reports of: being time-poor, not being able to make time for the modules, and not finding the modules enjoyable. These reports highlight difficulties students experienced in establishing time-management and learning priorities. It is also possible that students already possessed strong beliefs of their own knowledge gaps along with established methods for targeting these deficits, diminishing their perceived need to undertake the learning activity. Reducing the length of each module, perhaps using a random sample of five quiz questions rather than fifteen questions, may have provided students with a more efficient snap-shot of their abilities and appeared less daunting to their competing time-commitments. Students could then repeat the quiz as desired, with a new sample of questions appearing with each attempt. However, decreasing the quantity of content within the modules may also adversely affect its perceived usefulness by students in identifying knowledge deficits.

The need for guidance and direction, even within self-directed learning activities, has been expressed previously [16]. Students require learning structure, frequent feedback, and reassurance that they are learning about the prescribed topics in the correct manner. Additional learning supports that could have assisted uptake by students may have included a learning schedule, prompting students via electronic diary or email systems, to undertake the self-directed resource quiz at varying stages throughout the semester.

With assessment being a key driver to learning, making quiz participation a hurdle requirement may have provided a new student drive to undertake the self-directed resources, although this may have been counterproductive to the adult learning skills trying to be fostered. 
Results from the usage data and student surveys indicate a number of complex interactions occurring between the barriers and facilitators to student engagement. These interactions affect the uptake and perceived educational value for this form of self-directed resource. These interactions can be broadly categorised into drives, distractions, supports, and design factors. Drives, such as a student's desire for feedback, or a perceived need for revision in the resources applied study topic, contribute to students' increased use of a learning tool. On the other hand, distractions for students may include competing learning tasks, or students' competing academic and social foci. Resource supports include items that guide and facilitate student access and engagement. Further resource supports could have been applied in this instance by providing further information on how, when and why the modules were best utilised. Finally, the design of the learning tool itself is a contributing factor to student engagement. Even if the tool meets a student's requirements in terms of content, resources that are unattractive in presentation, duration and interactivity may deter student participation. Compared to the complex, colourful and entertaining computer programs and held device applications available, it is not surprising that our text-based module with minimal graphic content may have reduced students' participation. Careful design of self-directed learning activities, especially online modules, should remain a priority during development.

The interactions of these findings have been expressed as a conceptual model in Figure 1.

Figure 1. Conceptual model of student and resource considerations for maximising educational value.

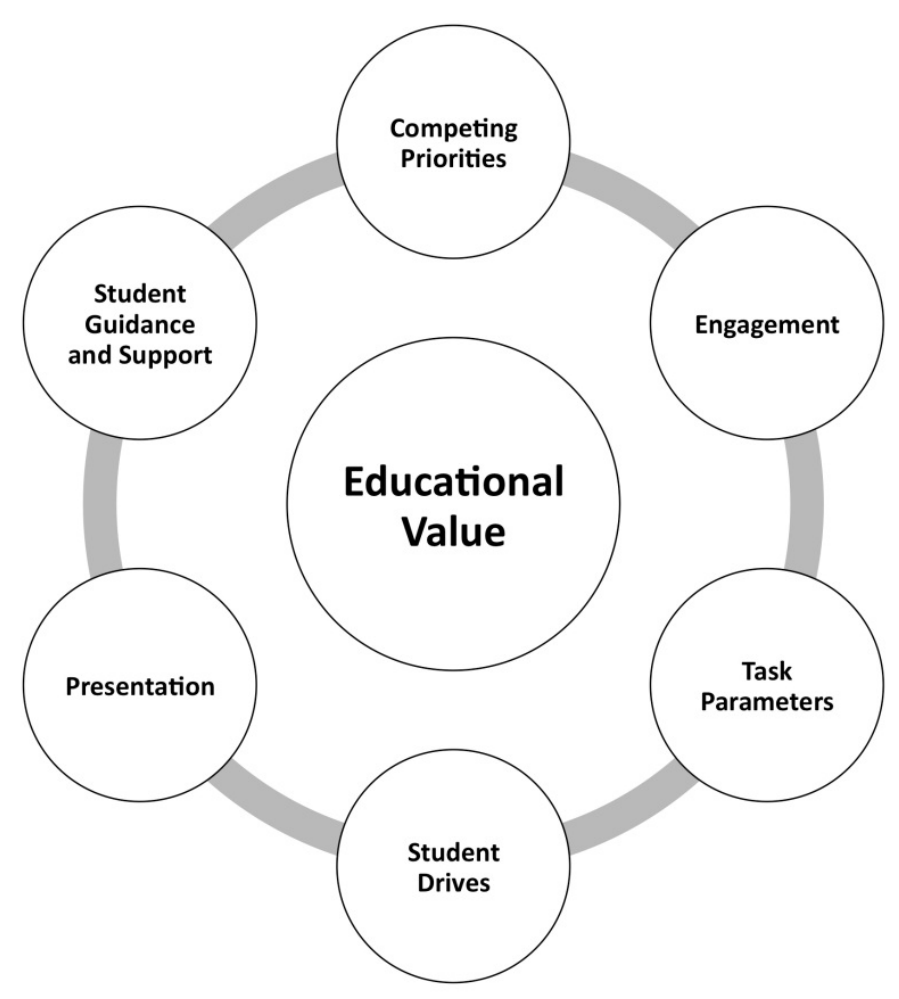

\section{Conclusions}

Students utilised the self-directed learning modules designed for supporting transition of skills and knowledge to the clinical environment in a predictable manner, with peak usage occurring before 
examinations. Students declared that the resources were valuable for identification of knowledge deficits and facilitated targeted revision. Less consensus existed on whether this benefit was superior to their own general reflection on abilities.

From the perspective of the Monash academic staff that constructed the resources, student uptake was considered unacceptably low for effectively supporting learning in the designated student cohort, and therefore an ineffective return for staff time-investment. The student survey data revealed a number of compelling concepts and recommendations that could have been incorporated into the design, implementation and support of these electronic resources to ensure optimum uptake and educational value.

The research team would make the following recommendations for other academics looking to create similar self-directed resources to support the further development of clinical-based skills for on-campus or clinical education based students:

- Keep the resource as time-efficient as possible. Allow students to revisit the task for further questions/participation, rather than deliver the resource in a daunting bolus.

- Consider learning supports to facilitate student engagement i.e. recommended learning schedule with email reminders.

- Ensure the task has a strong link to a clinical context.

- Consider what distracters can be removed from the student schedule at the time of implementation.

- Incorporate a mechanism for student feedback and refinement of the task activities.

- Evaluate student engagement via online learning system reporting features, to ensure adequate uptake and utilisation of the resource is being achieved.

- Use eye-pleasing presentation formats including images and digital multimedia where appropriate.

The educational value and return on staff investment of self-directed resources placed into the online environment cannot be assumed. Although these resources have the ability to support skill transition to the clinical environment, optimal educational value for self-directed resources requires careful consideration of both the resource design, and strategy for its implementation.

\section{Conflict of Interest}

The authors declare no conflict of interest.

\section{References}

1. Brockett, R.G.; Hiemstra, R. A conceptual framework for understanding self-direction in adult learning. In Self-Direction in Adult Learning: Perspectives on Theory, Research, and Practice; Routledge: London and New York, UK and USA, 1991

2. Candy, P. Self-Direction for Lifelong Learning: A Comprehensive Guide to Theory and Practice; Josey-Bass Publishers: San Francisco, CA, USA, 1991.

3. Jette, D.U.; Bertoni, A.; Coots, R.; Johnson, H.; McLaughlin, C.; Weisbach, C. Clinical instructors' perceptions of behaviors that comprise entry-level clinical performance in physical therapist students: A qualitative study. Phys. Ther. 2007, 87, 833-843. 
4. Erickson, M.L. Examining the presence of computer-assisted instruction in physical therapy education. J. Allied Health 2004, 33,255-266.

5. Spencer, J. Learning and teaching in the clinical environment. Br. Med. J. 2003, 326, 591-594.

6. Gordon, J.; Hazlett, C.; Ten Cate, O.; Mann, K.; Kilminster, S.; Prince, K.; O’Driscoll, E.; Snell, L.; Newble, D. Strategic planning in medical education: Enhancing the learning environment for students in clinical settings. Med. Educ. 2000, 34, 841-850.

7. Strohschein, J.; Hagler, P.; May, L. Assessing the need for change in clinical education practices. Phys. Ther. 2002, 82, 160-172.

8. Seabrook, M.A.; Woodfield, S.J.; Papagrigoriadis, S.; Rennie, J.A.; Atherton, A.; Lawson, M. Consistency of teaching in parallel surgical firms: an audit of student experience at one medical school. Med. Educ. 2000, 34, 292-298.

9. Singh, P.; Pan, W. Online education: Lessons for administrators and instructors. Coll. Student J. 2004, 38, 302-308.

10. Berger, N. Pioneering experiences in distance learning: Lessons learned. J. Manage. Educ. 1999, 23, 684-690.

11. Peacock, S.; Hooper, J. E-Learning in Physiotherapy. Physiotherapy 2007, 93, 218-228.

12. Hung, M.; Chou, C.; Chen, C.; Own, Z. Learner readiness for online learning: Scale development and student perceptions. Comput. Educ. 2010, 55, 1080-1090.

13. Liu, G.; Hwang, G. A key step to understanding paradigm shifts in e-learning: Towards context-aware ubiquitous learning. Br. J. Educ. Technol. 2009, 41, 1-9.

14. Rice, P.; Ezzy, D. Qualitative Research Methods; Oxford University Press: South Melbourne, Australia, 2009.

15. McNulty, J.A.; Sonntag, B.; Sinacore, J.M. Evaluation of computer-aided instruction in a gross anatomy course: A six-year study. Anat. Sci. Educ. 2009, 2, 2-8.

16. Raidal, S.L.; Violet, S.E. Preclinical students' predispositions towards social forms of instruction and self-directed learning: A challenge for the development of autonomous and collaborative learners. Int. J. Higher Educ. Educ. Plann. 2009, 57, 577-596.

(C) 2013 by the authors; licensee MDPI, Basel, Switzerland. This article is an open access article distributed under the terms and conditions of the Creative Commons Attribution license (http://creativecommons.org/licenses/by/3.0/). 